\title{
Familiarity with visual stimuli boosts recency bias in macaques
}

\author{
Nicolas Brunet ${ }^{\text {Corresp., } 1}$, Bharathi Jagadeesh ${ }^{2}$ \\ ${ }^{1}$ Department of Psychology and Neuroscience, Millsaps College, Jackson, Mississippi, United States \\ 2 Department of Physiology and Biophysics, University of Washington, Seattle, Washington, United States \\ Corresponding Author: Nicolas Brunet \\ Email address: brunenm@millsaps.edu
}

To probe how non-human primates (NHPs) decode temporal dynamic stimuli, we used a two-alternative forced choice task (2AFC), where the cue was dynamic: a movie snippet drawn from an animation that transforms one image into another. When the cue was drawn from either the beginning or end of the animation, thus heavily weighted towards one (the target) of both images (the choice pair), then primates performed at high levels of accuracy. For a subset of trials, however, the cue was ambiguous, drawn from the middle of the animation, containing information that could be associated to either image. Those trials, rewarded randomly and independent of choice, offered an opportunity to study the strategy the animals used trying to decode the cue. Despite being ambiguous, the primates exhibited a clear strategy, suggesting they were not aware that reward was given non-differentially. More specifically, they relied more on information provided at the end than at the beginning of those cues, consistent with the recency effect reported by numerous serial position studies. Interestingly and counterintuitively, this effect became stronger for sessions where the primates were already familiar with the stimuli. In other words, despite having rehearsed with the same stimuli in a previous session, the animals relied even more on a decision strategy that did not yield any benefits during a previous session. In the discussion section we speculate on what might cause this behavioral shift towards stronger bias, as well as why this behavior shows similarities with a repetition bias in humans known as the illusory truth effect. 


\section{Familiarity with visual stimuli boosts 2 recency bias in macaques.}

3 Nicolas M. Brunet ${ }^{1}$ and Bharahti Jagadeesh ${ }^{2}$

$4{ }^{1}$ Millsaps College, Department of Psychology and Neuroscience, Jackson, MS 39210, USA.

5 2University of Washington, Department of Physiology and Biophysics, Seattle, WA 98195, USA.

6

7 Address correspondence to Nicolas Brunet, Millsaps College, $1701 \mathrm{~N}$ State, Jackson MS 39210, USA.

8 E-mail: brunenm@millsaps.edu. Tel: +1 6019741389.

9

10 Running title: Decoding stimuli reveals repetition bias in macaques. 


\section{Abstract}

13 To probe how non-human primates (NHPs) decode temporal dynamic stimuli, we used a twoalternative forced choice task (2AFC), where the cue was dynamic: a movie snippet drawn from an animation that transforms one image into another. When the cue was drawn from either the beginning or end of the animation, thus heavily weighted towards one (the target) of both images (the choice pair), then primates performed at high levels of accuracy. For a subset of trials, however, the cue was ambiguous, drawn from the middle of the animation, containing information that could be associated to either image. Those trials, rewarded randomly and independent of choice, offered an opportunity to study the strategy the animals used trying to decode the cue. Despite being ambiguous, the primates exhibited a clear strategy, suggesting they were not aware that reward was given non-differentially. More specifically, they relied more on information provided at the end than at the beginning of those cues, consistent with the recency effect reported by numerous serial position studies. Interestingly and counterintuitively, this effect became stronger for sessions where the primates were already familiar with the stimuli. In other words, despite having rehearsed with the same stimuli in a previous session, the animals relied even more on a decision strategy that did not yield any benefits during a previous session. In the discussion section we speculate on what might cause this behavioral shift towards stronger bias, as well as why this behavior shows similarities with a repetition bias in humans known as the illusory truth effect.

\section{Introduction}

The cognitive process of selecting among alternatives, hoping for the most favorable outcome, is not unique to humans. Many studies that investigate biases and decision-making strategies in humans have been replicated with non-human animals. Some of those cognitive biases have been observed in various animal species (ranging from pigeons to primates), suggesting a strong evolutionary link. One example is hyperbolic discounting, which reveals a preference for small rewards that occur sooner, rather than larger ones that occur later. See (Vanderveldt, Oliveira, \& Green, 2016) for a review. Another example is the serial position effect, characterized by the typical U-shaped relationship between an item's position in a list and the probability to recall it; this effect is demonstrated for pigeons (Santiago \& Wright, 1984), rhesus macaques (Sands \& Wright, 1980), and other species. The hot hand fallacy or the "belief" that experiencing successful outcome leads to a greater chance of success in further attempts was reported in a study with rhesus macaques (Blanchard, Wilke, \& Hayden, 2014). Also the ability to make rational choices, using paradigms that are even challenging for humans, such as the Monty Hall dilemma - a 
statistical illusion - have been studied in birds (Herbranson \& Schroeder, 2010), and primates (Klein, Evans, Schultz, \& Beran, 2013).

Ambiguous stimuli have been proven to be an effective strategy to probe the behavior and cognitive bias in animals. Typically, an animal learns to discriminate between stimuli that predict positive consequences and stimuli that predict a negative outcome. See Roelofs et al. for a comprehensive review (Roelofs, Boleij, Nordquist, \& van der Staay, 2016). Once the animal masters the task, ambiguous stimuli are introduced that lie between the original stimuli. The hypothesis is that the animal's "mood" will bias the choice following an ambiguous test stimulus. If its mood is positive, then it will classify the stimulus as positive. Correspondingly, if the animal's mood is negative, it will classify the stimulus as negative. This approach has been used to show that laboratory rats in unpredictable environments had a more pessimistic attitude than rats in predictable environments (Harding, Paul, \& Mendl, 2004), and that dogs who exhibit high levels of separation-related behavior have a more negative underlying mood (Mendl et al., 2010). The approach is now considered as a valuable indicator of animal wellbeing, applicable to many species, ranging from honey bees (Bateson, Desire, Gartside, \& Wright, 2011) to non-human primates (Burman, Parker, Paul, \& Mendl, 2009).

In this study, the researchers use ambiguous stimuli, not to test the mood of the animal, but to gain insight about how it visually perceives the ambiguous stimuli. A method often used to measure the subjective experience of primates is the two-alternative forced choice (2AFC) task. Typically, for each trial, an auditory or visual stimulus is presented, which then needs to be categorized into the correct class. The subject does so by selecting one of two possible options. When the answer is correct, animals usually receive food pellets or a juice reward. Similar to the cognitive bias studies to assess mood, described above, we insert "impossible trials" where the cue is ambiguous once the animal masters the task. The ambiguous cue in this case is derived from two cues used for the unambiguous trials, where each is linked to a different outcome. To mask the unsolvable nature of those trials, ambiguous and unambiguous trials are mixed, and ambiguous trials rewarded at random (50\%), regardless of the answer.

For this study, we were particularly interested in how familiarity with visual stimuli influences decision and whether this would result in a change of behavioral bias. Familiarity with visual stimuli modulates neural processing along the ventral visual pathway. In humans, visual evoked potentials in response to familiar stimuli are larger than those recorded in response to new stimuli (Tanaka \& Curran, 2001). Also in rhesus macaques, signals retrieved from electrodes placed over the occipital cortex (Peissig, Singer, Kawasaki, \& Sheinberg, 2006) and the temporal lobe (Anderson, Mruczek, Kawasaki, \& Sheinberg, 2008) reveal stronger evoked potentials for familiar compared with novel stimuli. Erickson et al. show that neuronal preferences for pairs of nearby neurons, in inferotemporal cortex, differ greatly for novel stimuli, but become more 
82 similar after experience with the stimuli (Erickson, Jagadeesh, \& Desimone, 2000). To probe 83 whether behavior would be susceptible to the familiarity of the visual stimuli in a task where 84 familiarity with the stimuli (or lack thereof) is irrelevant for performance and reward, we 85 designed a 2AFC task where the cue was a dynamic visual stimulus. Unbeknownst to the animals, 86 a subset of trials, rewarded at random, were ambiguous since there was no correct nor incorrect 87 answer. In order for our approach to work, it was crucial that the animals, in response to the 88 "impossible trials", displayed a non-random behavior consistent with a decision strategy. The 89 results of the first sessions did show that both animals indeed elicited the same biased behavior, suggesting genuine attempts to maximize reward. Satisfied with the preliminary results, we shifted to the main objective of our study: investigate whether reusing the same stimuli (now familiar to the subject) would result in a behavioral shift. The three possible outcomes are the 93 following:

(1) a weakening of the bias, with stimulus familiarity.

This outcome suggests that the animals learned that, for the subset of trials where ambiguous cues are used, reward is unrelated to behavior. Not receiving any benefit from making a mental effort to make a decision, could result in random behavior; alternatively, the primates might attempt to minimize efforts by selecting an image based upon its spatial position (for instance, always the one displayed at the bottom), rather than its contents.

(2) a preservation of the bias, with stimulus familiarity.

If the way that the NHPs respond to familiar stimuli is the same as to novel stimuli, then it might indicate that their perception of the stimuli has not been changed. In other words, the mechanisms of how those stimuli are processed temporally are not affected by the familiarity of the stimuli.

(3) a strengthening of the bias, with stimulus familiarity.

This is the most counterintuitive outcome, because the primates are neither rewarded nor trained to display such behavior. A form of long-term adaptation (LTA), potentially associated with learning and/or memory, might take place during the session where the stimuli are novel. If so, then the temporal processing of those stimuli will be affected when used again during a later session, consistent with the stronger visual evoked potentials observed in human and non-human primates in response to familiar stimuli (Anderson et al., 2008; Peissig et al., 2006; Tanaka \& Curran, 2001). 


\section{Materials and Methods}

114 Subjects

115 Data were obtained from two adult male rhesus monkeys (7-10 kg). Animals were housed in 116 standard cages in the Washington Primate Research Center, with pair housing when possible (Liu, 117 Murray, \& Jagadeesh, 2009). Monkeys B. and S. were trained, acquired, and raised under the 118 same protocol (\# 3275-01) as monkeys L. and G. (Akrami, Liu, Treves, \& Jagadeesh, 2008; Liu et 119 al., 2009). Prior to this study, the animals were well trained in versions of the 2 -alternative forced 120 choice design used in this experiment (Liu \& Jagadeesh, 2008). During each session, NHPs were 121 positioned in a booth, and performed a two alternative forced choice-delayed match to sample 122 task (2AFC). Each trial consisted of a series of events, displayed on a computer monitor, and 123 ended with a response in the form of a saccade (see Experimental Design). The stimuli were 124 displayed using CORTEX, a program for neural data collection and analysis developed at the NIH 125 (Bethesda, MD). Both NHPs were implanted with a head restraint prosthesis, used to limit 126 movement of the head during data collection, and a scleral eye coil to monitor eye position (DNIm 127 Newarkm DE, USA; (Judge, Richmond, \& Chu, 1980)). To induce motivation for the cognitive task, 128 freely available water was limited the day before the experiment, and fluids (water or juice drops) 129 used as a reward following a correct trial. All animal handling, care and surgical procedures were 130 performed in accordance with guidelines established by the $\mathrm{NIH}$, and approved by the 131 institutional Animal Care and Use Committee (IACUC) at the University of Washington (Protocol 132 number 3275-01).

\section{Stimuli and experimental design}

134 For each session, one pair of photos was selected from a picture database containing images of 135 everyday objects such as faces, plants, animals, landscapes and buildings (see Fig. 1A for some 136 examples). All images were $90 \times 90$ pixels and were drawn from a variety of sources, including image databases, and personal photo libraries. Stimuli were presented on a computer monitor

138

139

140

141

142

143

144

145

146

147

148 with $800 \times 600$ resolution and a refresh rate of $100 \mathrm{~Hz}$. At the viewing distance used, images subtended $4^{\circ}$ of visual angle. All visual stimuli used for that session were then derived from those images. The visual stimuli were either familiar to the subject (used during a previous session) or novel (never used before).

The sequence for each trial was the same (see Fig. 1C): after the monkey foveated a fixation point for a variable time between 250 and 500 ms within an invisible fixation window that was 4 degrees in diameter and centered around the fixation point, a visual cue was presented. The cue was displayed for $500 \mathrm{~ms}$, and either static or dynamic (see below), and its location and size the same as for the invisible fixation window. After the cue disappeared, the fixation point was still turned on for a variable delay time between 700 and $1200 \mathrm{~ms}$, and after the choice pair was displayed, for another variable period of $400-700 \mathrm{~ms}$. Only then was the fixation point turned off, 
149 signaling the NHP that it was allowed to make a choice. The NHP could express its preference by 150 making a saccade towards either image A or image B (together forming the choice pair). The 151 monkey received a reward for selecting the image (either $A$ or $B$ ), that best matched the cue.

152 To avoid spatial bias, the order of the choice pair, located 5 degrees to the left of the fixation 153 point was randomized: Image A was 5 degrees either up or down, and image B respectively 5 154 degrees down or up with respect to the fixation point (see Fig. 1C).

155 For trials where the cue was static (either image A or image B) reward followed the selection of

156

157

158

159

160

161

162

163

164

165

166

167

168

169

170

171

172

173

174

175

176

177

178

179

180

181

182

183 the corresponding image from the choice pair (delayed match-to-sample; see Fig. 1C). For some trials, however, a dynamic cue was used: a movie snippet of 5 frames, drawn from an 11-frame long movie that was generated by morphing image A (frame 1) slowly into image $B$ (frame 11; see Fig. 1B, blue and pink boxes). The images were morphed by using MorphX (http://www.norrkross.com/software/morphx/morphx.php), a program for morphing between two photographic images. The dynamic cues, consisting of either the first or last five frames of the larger movie, were displayed for $500 \mathrm{~ms}$ (100 ms per frame) and presented either in forward or reverse order. Trials with dynamic cues were rewarded when the NHP selected the target (from the choice pair) that was also presented in the cue. More specifically, trials where the cue was a movie snippet consisting of frames 1-2-3-4-5 or 5-4-3-2-1, led to reward when the animal selected image $A$ (equal to frame 1 ), whereas trials where the cue was a movie snippet consisting of frames 7-8-9-10-11 or 11-10-9-8-7 led to reward when the NHP selected image $B$ (equal to frame 11).

For a subset of the trials, the dynamic cue was ambiguous, consisting out of the five frames drawn from the middle of the 11-frame movie, thus containing neither image A nor image $B$ (see Fig. $1 B$, green box). The ambiguous cue was presented either in forward (frames 4-5-6-7-8) or reverse direction (frames 8-7-6-5-4). For those ambiguous trials, where there was no correct answer, the animals were rewarded randomly at a $50 \%$ chance rate upon completing the trial.

For some of the sessions, the dynamic cue was "weighted". We introduced this condition after we noticed - based upon preliminary results - that the NHPs were more likely to extract information from the end of a dynamic cue than from the beginning (see results). We were interested in whether weighting the movie towards the beginning, by removing the penultimate frame (frames with a red crossed box, in the example shown in Fig. 1B), would change the behavior of the NHPs. The intervention, resulting in a $100 \mathrm{~ms}$ long gap during the display of the cue, however made perceptually almost no difference, and consequently did not affect the behavior of the animals (as reported in the result section).

Sessions were organized in blocks of 60 trials. The first block contained only unambiguous static cues (see Fig. 1B, row 2 for an example), which was meant to motivate the subject and to make 
184 it familiar with the stimulus pair before confronting it with trails where the cues were dynamic. 185 Subsequent blocks, also 60 trials long, consisted of all 10 conditions, randomly mixed. Those are 186 the two static conditions (Fig. 1B, row 2, the four unambiguous dynamic conditions (Fig. 1B, 187 movie snippets in blue and pink boxes), two ambiguous dynamic conditions (Fig. 1, B, green box) 188 always followed by reward, and the same two dynamic conditions, but never followed by reward. 189 We collected minimum five blocks of data for each session, yielding at least 96 trials where 190 ambiguous cues were used. We collected data from 141 sessions for monkey B. and 269 sessions 191 for monkey S., totaling more than 200,000 trials.

192

193

Data analysis

194

All analyses, including averages, error estimates, and statistical significance were calculated using Matlab, version R2015a; Figures were generated using Matlab, version R2015a, and Adobe 196 Illustrator CS6. Averages are given as unweighted mean \pm SE.

197

To quantify the results obtained for this study, we used two equations.

198

The first equation is used to calculate the Preference Index (PI), a metric that quantifies temporal biases which we termed recency and primacy (see results). PI is calculated as follows:

$200 \quad P I=\left(\frac{R-P}{R+P}\right)$

201

202

203

204

205

206

207

208

209

210

211

212

213

where $\mathrm{R}$ and $\mathrm{P}$ represent the number of ambiguous trials within a given session where the NHP respectively selected the image most resembling the end (Recency) or beginning (Primacy) of the dynamic cue (see Experimental design and Fig. 2, green box).

A second equation was used to quantify the behavioral shift $\left(\Delta_{\mathrm{PI}}\right)$, between two sessions (S1 and S2) where the same set of visual stimuli were used. We denote S1 as the session where a given set of stimuli was used for the first time and $S 2$ as the session where the same set was used again, thus for a second time. The $\Delta_{\mathrm{PI}}$ for a pair of S1 and S2 is obtained as follow:
$\Delta_{P I}=P I_{S 2}-P I_{S 2}$
(eq. 2)

where $\mathrm{PI}_{\mathrm{S} 1}$ and $\mathrm{P}_{\mathrm{IS} 2}$ are the $\mathrm{PI}$ values (see eq. 1) calculated for respectively S1 and S2.

\section{Results}

\section{Discrimination between two visual stimuli, using static and dynamic cues}

Before acquisition of the behavior data used for this study, two NHPs were first trained in the 2AFC task using only static cues (Delayed matching-to-sample). Once the NHPs routinely 
214 performed at high levels of accuracy (> $90 \%$ correct), we also included 5 -frame long dynamic

215 cues (see methods), drawn from a larger 11-frame long movie generated by morphing image $A$

216 (frame 1) stepwise into image B (frame 11).

217 Once the NHPs also mastered trials with dynamic cues ( $>90 \%$ correct), we switched from training 218 to working mode, and composed a complete new set of image pairs, from which we selected a 219 single pair for each session (see Fig. 1A for some example pairs).

220 The animals performed well on trials with dynamic cues, whether the target frame was included

221

222

223

224

225

226

227

228

229

230

231

232

233

234

235

236

237

238

239

240

241

242

243

244

245

246

247

248 at the beginning (Fig. 2A and 2B, purple bars; forward direction) or end (green bars; reverse direction) of the dynamic cue, as illustrated by the success rates: $86.0 \pm 1.1 \%$ and $86.3 \pm 1.0$ (monkey B); and $93.6 \pm 0.7$ and $94.1 \pm 0.6 \%$ (monkey S) for respectively forward and reverse playing directions. A two sample t-test comparing the means of the success rates computed for each playing direction revealed that the playing direction of the cue had no effect on performance (monkey B.; $\mathrm{p}=0.80 ; \mathrm{N}=107$ and monkey $\mathrm{S} ; \mathrm{p}=0.40 ; \mathrm{N}=131$ ). As expected, the NHPs were not able to figure out a strategy allowing them to receive more reward than predicted by chance (Fig. 2A and B, gray bars) for trials with ambiguous cues, demonstrating that the reward was truly random: $49.5 \pm 0.5 \%$ for monkey $B$., and $49.9 \pm 0.3 \%$ for monkey $S$. To examine whether performance changed over the course of a session, which might signal fatigue, we also computed the proportion of rewarded trials as a function of trial number for a given condition. To that extent, we normalized each session with respect to the total number of trials. The results, based upon an average of all sessions (Fig. 2C and 2D) suggest that the primates did neither change behavior nor strategy with respect to any of the conditions during the course of a session.

Not displaying the penultimate frame (weighted cues; see methods) caused a small interruption in the sequence, and potentially a perceptual shift towards the beginning of the dynamic cue (see methods). The intervention, however, did affect neither behavior nor performance as illustrated by a two sample t-test comparing the means of the success rates for sessions of each condition: there was no statistical difference between sessions using weighted versus unweighted movies for the forward direction ( $p=0.06$ for monkey $B$. and $p=0.14$ for monkey $\mathrm{S}$.) nor for the reverse direction ( $p=0.21$ for monkey $B$. and $p=0.76$ for monkey $S$.).

\section{Recency and primacy effects}

Because the performance of the NHPs for trials with informative cues was very satisfactory, not sensitive to the direction of the dynamic cue, and consistent over the course of a session (see Fig. 2), we were confident that the NHPs, even when facing ambiguous cues, would show nonrandom behavior by continuing to apply the learned rules for making a "correct" response. To probe the behavior with respect to those "impossible" trials, the ultimate aim of this study, we will from here onwards only consider the data from those trials. Moreover, for this subset of the 
249

250

251

252

253

254

255

256

257

258

259

260

261

262

263

264

265

266

267

268

269

270

271

272

273

274

275

276

277

278

279

280

281

data, we will no longer focus on correct versus non-correct identification of the target which was determined random and therefore not informative, but instead on the interpretation of the cue by the animal. Would it rely, trying to identify the "correct" target, on the information extracted from the beginning (more similar to one image from the choice pair), or rather from the end (more similar to the other image) of the dynamic cue? To quantify the weight of each of those effects, we calculated a Preference Index ( $\mathrm{Pl}$; see eq. 1 in methods). This metric yields a value between -1 and +1 for each session. A value smaller than 0 reveals that for this session the primate was more likely to engage in primacy bias, while a value greater than 0 indicates that the recency effect prevailed. It should be noticed that the terms "primacy" and "recency", for this study, are not used in the same way as for serial position effect studies (Murdock, Bennet B., 1962), where they refer to the tendency of an individual to respectively best recall the first and last items of a list.

Because weighting of the stimuli had no significant effect on the PI values, we merged both conditions for further analysis. Computation of the mean PI value, averaged across all 141 sessions from monkey B. and all 269 sessions from monkey S. revealed that both primates engaged in behavior that slightly, but consistently, tilted towards recency bias (Fig. 3A and 3B). The averaged PI computed for monkey B. was $0.08 \pm 0.01$ (inset Fig. $3 \mathrm{~A}$ ) and $0.09 \pm 0.01$ for monkey S. (inset Fig. 3B). Although the effect size appears small, it was statistically very significant for each animal, as demonstrated by using a non-parametric sign test yielding $p$-values of $1.7 \mathrm{x}$ $10^{-6}$ and $3.2 \times 10^{-9}$ for monkeys B. and S. respectively. This tendency towards recency is also illustrated by the distribution of the PI values (Fig. 3A and 3B), where the bin with value 0 (marked by a vertical black line) represents sessions where the recency and primacy effects were equally balanced.

It is possible that towards the end of the session NHPs, with respect to ambiguous trials, would try "less hard" to find the "correct answer". If so, then the PI value would converge towards 0 over the course of a session, consistent with random and effortless response selection. Taking the same approach as we did to study behavior with respect to unambiguous trials over the course of a session (Fig. 2C and D), we also computed the PI value as a function of normalized trial number. The results (Fig. 3C and 3D) suggest that also in this case, the behavior of the NHPs did not change across the duration of a session.

Taken together, the results shown in Fig. 3 suggest that the NHPs, despite receiving reward at random, did not choose randomly but used a choice strategy as illustrated by a small but very significant recency effect.

PeerJ reviewing PDF | (2019:05:38089:3:0:NEW 20 Oct 2019) 


\section{The effect of stimulus familiarity on behavior bias}

283 To study the effect of familiarity of the visual stimuli, we examined whether behavior exhibited

284

285

286

287

288

289

290

291

292

293

294

295

296

297

298

299

300

301

302

303

304

305

306

307

308

309

310

311

312

313

314

315

316 during a session where a given pair of images was novel (denoted S1) shifted when those same stimuli were used again, later, during a second session (denoted S2). Across both NHPs, we identified 163 instances where the same stimuli were used for two different sessions by the same animal ( $2 \times 49$ sessions from monkey B. and $2 \times 114$ sessions from monkey S.). The averaged PI value of all S1 sessions (Fig. 4A and 4B) was positive for both animals $(0.008 \pm 0.024$ and $0.062 \pm$ 0.015 for monkeys B. and S. respectively), consistent with recency bias. This was also the case for all S2 sessions $(0.130 \pm 0.023$ and $0.099 \pm 0.017$ for monkeys $B$. and $\mathrm{S}$. respectively). A paired $\mathrm{t}$ test between the PI values of S1 and S2 from each pair, however, revealed that this recency bias strengthened significantly with stimulus familiarity, for both monkey $B$. $(p=0.0001)$, and monkey $S$. $(p=0.041)$. This effect of familiarity is further visualized in Fig. $4 B$, where each of 163 data points (green for monkey B and light purple for monkey S.) indicate a pair of sessions (S1 and S2) where the same stimuli were used. The coordinates of each data point correspond with the PI values of S1 and S2.

Those results are counterintuitive, because it was expected that the NHPs, after gaining experience with the stimuli, would begin to unmask the dubious nature of the ambiguous trials, and start selecting the "target" more randomly, resulting in a PI value closer to 0 (the value associated with chance). Instead, reusing the same stimuli strengthened the recency effect, suggesting that the primates, instinctively, increased the use of a strategy that has not proven to pay off.

This observation gives rise to a new question: "is the lag time between a pair of sessions (S1 and S2) critical for the observed phenomenon?"

To answer that question, we computed $\Delta_{\mathrm{PI}}$ (see methods for details), between any pair of sessions where a given set of stimuli was used for respectively the first (S1) and second time (S2). This yielded a single value for each of the 163 pair of sessions revealing whether familiarity with a given set of stimuli would not affect behavior (values close to 0 ), would induce a shift towards primacy bias (negative value), or cause a shift towards recency bias (positive value).

To take the elapsed time (measured in days) between S1 and S2 into account, we divided the 163 pairs of sessions into 3 bins (Fig. 4D). The first bin contained all the pairs of sessions where S1 and S2 were separated by 3 days or less (12 pairs for monkey B; 48 pairs for monkey S.); the second bin all pairs of sessions separated by 4 to 6 days (18 pairs for monkey B. and 28 pairs for monkey S.); and a third bin for sessions separated by more than 6 days (19 pairs for monkey B. and 38 pairs for monkey S.). Interestingly, a paired t-test between the S1 and S2 sessions revealed that the behavioral shift is not statistically significant (for neither NHP) for the pairs grouped in 
317 the first bin, but significant (monkey S.; $p=0.030$ ) or even extremely significant (monkey B.; $318 p=0.0003)$ for the pairs that make up the second bin. The effect then seems to fade away again 319 for sessions separated for more than 6 days (3rd bin), still significant in monkey B. $(p=0.034)$ but 320 not in monkey $\mathrm{S} .(p=0.313)$.

321 If the primates over the course of many sessions, recorded over a period of 10 months, exhibited 322 increasingly more recency behavior, then the observed behavioral shift (Fig. 4 A-C) could simply

323 be explained due to the fact that S2 was recorded at a later date than S1, and not because of 324 familiarity with the stimuli. Plots of PI versus recording day (Fig. 5A and 5B), however, show that 325 the animals did not change their behavior over the course of $\sim 300$ days of data collection. The 326 slope of the linear regressions (black lines in Fig. 5A and 5B) are not statistically different from 327 zero ( $p=0.09$ for monkey B., and $p=0.96$ for monkey $\mathrm{S}$.).

328 Because those unambiguous trials were rewarded at random, it is not impossible that for some 329 sessions, a given strategy (for instance recency) was rewarded more often, or less often, than

330

331

332

333

334

335

336

337

338

339

340

341

342

343

344

345

346

347

348

349

350 what would be expected by chance. To investigate whether our results could be explained by a reinforcement history, we plotted the behavior shift between S1 and S2 $(\Delta \mathrm{PI})$ as a function of how often a given strategy, primacy (Fig. 5C) or recency (Fig. 5D), was rewarded during performance of S1. The insets of each figure shows how the linear regression of the data would look if there was a strong effect of reinforcement history. For instance, always (never) receiving reward for choosing recency during S1 would trigger the animal to rely more (less) on recency during S2, which would then result in a more positive (negative) $\Delta$ PI (inset Fig. 5D). The analysis (Fig. 5C and D), however, demonstrates that our results cannot be explained by reinforcement history: the slopes of the linear regressions (green line for monkey B. and purple line for monkey S.) in Fig. $5 \mathrm{C}$ are not statistically different from zero ( $p=0.55$ for monkey B., and $p=0.27$ for monkey S.); this is also the case for the regressions shown in Fig. 5D ( $p=0.19$ for monkey B., and $\mathrm{p}=0.92$ for monkey $\mathrm{S}$.)

Taken together, the results, shown in Fig. 4 and 5, suggest that the cognitive processes that took place while the visual stimuli were used for the first time, altered behavior during a later session when those same stimuli were used again. Interestingly, the influence appears to be mild when familiarity with the stimuli is only established recently, but strong when stimuli used for the first time are used again after 4 to 6 days. Unsurprisingly, the effect decreased with longer time between both sessions.

\section{Discussion}

When NHPs, trained to interpret a visual cue, were faced with cues both dynamic and ambiguous, they relied more on the information contained at the end than at the beginning of the cue. Using 
351 nomenclature borrowed from cognitive memory studies, we labeled this behavior recency bias.

352 Although our experimental setup differs greatly from those used for serial position studies, the 353 observed recency bias is consistent with that reported for a variety of serial position studies 354 under a variety of conditions (Bonk \& Healy, 2010; Bonnani, 2007; Farrand, Parmentier, \& Jones, 355 2001; Tremblay, Parmentier, Guérard, Nicholls, \& Jones, 2006).

356 Because those results are neither unexpected nor surprising, and in agreement with the literature 357 they, a fortiori, validate the next level of analysis resulting in our most intriguing finding; i.e., that 358 the observed cognitive bias is strengthened by the familiarity with the stimuli.

359 Non-human primates quickly become experts (Feng, Holmes, Rorie, \& Newsome, 2009) in 360 minimizing effort while optimizing reward, for tasks they have been trained in during weeks or 361 months. We were, however, not concerned that the primates would stop making a mental effort 362 by selecting a target at random in response to the "impossible" trials. This because the animals 363 were trained under conditions that lead to high-probability reward, encouraging them to figure out 364 the rule, or associative contingencies, that leads to reward. The results show, indeed, that the 365 primates - for the "impossible" trials - did not choose at random, but genuinely selected an 366 option anticipated to leading to reward. The results demonstrate that the primates, when 367 choosing one of two possible alternatives are more likely influenced by the information provided 368 at the end of an ambiguous dynamic cue (recency), than at the beginning (primacy).

369 From a behavioral perspective, the robust increase with stimulus familiarity in recency bias is 370 highly counterintuitive: it confirms that the NHPs - despite having rehearsed with those same 371 stimuli before - not only failed to uncover that the reward for unambiguous trials was non372 differential, but relied even more upon a strategy that does not yield benefits.

373 One obvious explanation for those results is that one of the two parent stimuli was more 374 appealing to the primate. This explanation, however, needs to be discarded because ambiguous 375 dynamic cues were controlled for direction so that choices influenced by a "favorite" stimulus 376 would cancel each other out with respect to primacy and/or recency bias.

377 More than 70 years ago, Skinner demonstrated that pigeons are susceptible to superstition, by 378 showing that they behave as if there were a causal relationship between behavior and reward 379 (Skinner, 1948). Although we cannot prove or disprove that superstitious behavior explains our 380 results, we do not think that this is the case. First of all, recency behavior did not strengthen 
381

382

383

384

385

386

387

388

389

390

391

392

393

394

395

396

397

398

399

400

401

402

403

404

405

406

407

408

409

410

411

across sessions (Fig. 5A,B); and second, the lack of correlation between how trials were rewarded during S1 and behavior during S2 (Fig. 5C,D) makes it unlikely that the animals adapted a behavior reminiscent of superstition.

A more plausible explanation for the observed familiarity effect is representational momentum (Nijhawan, 1994), an error in visual perception where an observer believes that a moving object is further along its pathway than it is in reality. In humans, the error is thought to be caused by two factors: the delay in visual processing (Lappe, Krekelberg, \& Lappe, 2000) and intrinsic knowledge of the laws of physics. For instance, if we follow a car speeding along the highway, with our eyes, then our brain will compensate (too much) for the delay in visual processing by anticipating where the car will be instances later. Interestingly, this perceptual error has not only been demonstrated for events hardwired in the perceptual system of our brain (Shepard, 1984), but also for dynamic scenes where the chain of events is expected. It is thus possible that this bias can be artificially induced, for instance, by repeated exposure to dynamic images whereby one object is turned into another. After frequent exposure, such an artificial sequence might be perceived as a "natural" chain of events. An older study demonstrated that people had greater difficulty to discriminate between two frames if they were not used to seeing those in sequence (Freyd, 1983). A chain of events implies movement, and where there is movement, representational momentum effects might be applicable. Monkeys cannot rationalize what they see as humans do, and therefore might incorporate the morphed directional dynamic images as a physical and natural transformation. If so, then representational momentum might explain the reported familiarity effect. In other words, after having been familiarized with the stimuli during a previous session, the NHP brain can now anticipate the "next" frame or frames (to compensate for the visual delay) during the presentation of the ambiguous dynamic cue, even if the "next" frame will not be displayed. This anticipation effect might thus weight visual perception in favor of "recency", and explain why we observed an increase of recency bias with familiarity with the stimuli.

Changes at the molecular level induced by novelty or familiarity with visual stimuli have been reported for rats (Zhu, Brown, \& Aggleton, 1995) and mice (Kissinger, Pak, Tang, Masmanidis, \& Chubykin, 2018). Other groups observed an increase in evoked potential with familiarity of the visual stimulus (Anderson et al., 2008; Peissig et al., 2006); Kissinger et al. (Kissinger et al., 2018) also reports an increase of theta oscillations with stimulus familiarity. At the behavior level, 
412 stimulus repetition has been observed to improve perception and performance. Grill-Spector et 413 al. (Grill-Spector, Henson, \& Martin, 2006) have reviewed the study of the neuronal mechanisms 414 involved in stimulus repetition. One leading hypothesis, at the system level, is that repetition 415 sharpens neural representation, which results in the increase of neuronal synchronous 416 oscillations (Brunet et al., 2014). Whether changes in molecular expression, modulation of visual 417 evoked potentials, and/or changes in neural rhythms triggered by stimulus familiarity leads to 418 the modulation of temporal dynamics, and in turn results in altered visual perception that 419 accounts for our results, requires further investigation.

420 Our results also show that the behavior shift induced by stimulus familiarity is optimal when a 421 set of stimuli is used again after 4 to 6 days (Fig. 4D). Why it peaks for this particular latency is 422 not clear since the timeline does not correspond with any known neurological or psychological 423 process. One possible explanation is that underlying changes in gene expression, induced by 424 novel stimuli (stimuli presented during session S1) require several days to reach full potential. If 425 so, then those molecular changes would reverse back to baseline as time goes by, consistent with 426 the results shown in Fig. 4D (third bin).

427 Finally, we noticed that the results of our study show remarkable similarities with a phenomenon 428 known as the Illusory Truth Effect. This bias, well studied in humans, shows that repeated 429 statements are more likely judged as true than unrepeated statements (Begg, Anas, \& Farinacci, 430 1992; Dechêne, Stahl, Hansen, \& Wänke, 2010). The leading hypothesis is that the repetition 431 causes processing fluency, which in turn drives the illusory truth effect (Fazio, Brashier, Keith 432 Payne, \& Marsh, 2015); thus not unlike the facilitation of neuronal processing caused by the 433 repetition of visual stimuli (Brunet et al., 2014; Gotts, Chow, \& Martin, 2012). In a study where 434 participants were scanned with $\mathrm{fMRI}$ while rating the truth of unknown statements, one brain 435 region, the perirhinal cortex, was shown to interact between repetition and ratings of perceived 436 truth. Interestingly, activity in that area only increased for statements that were presented to the 437 participant before being scanned (and thus repeated) but not for new ones (Wang, Brashier, 438 Wing, Marsh, \& Cabeza, 2016). Intriguingly, rhesus macaques lesioned in this same area (the 439 perirhinal cortex) exhibit familiarity judgment deficits, requiring more exposure to objects before 440 they are able to judge them as familiar compared to control animals (Weiss, Guo, Richardson, \& 441 Bachevalier, 2017). In both cases (illusory truth effect and the here reported behavioral shift) the 442 subject is unaware that repeated exposure leads to the strengthening of an unproven concept 443 (reliability of statements in humans, and decision strategy in monkeys). Whether those 
444 apparently different biases, share common neural correlates appeals for further investigation.

445 The illusory truth effect explains our propensity to accept fake news as true (Lazer et al., 2018),

446 which has been called a threat to democracy by Barack Obama in 2016. An animal model that

447 mimics this cognitive bias could thus fuel future studies that address this bias that has such a far-

448 reaching implication.

449

450 Conclusion

451 This study reports the following findings: (1) NHPs can be trained to excel in a 2AFC task that 452 features static as well as dynamic cues. (2) NHPs do not choose haphazardly when faced with 453 trials where the visual cue is ambiguous and the reward given at random; they instead use a 454 choice strategy that differs from non-random behavior. For those "impossible trials", the NHPs 455 based their response on the information provided at the end of the dynamic cue (recency) rather 456 than at the beginning of the cue (primacy). The observed recency bias was small but consistent 457 and very significant. (3) Interestingly, the recency bias was stronger for a session where a set of 458 stimuli was used a second time compared with that of the session where those stimuli were used 459 for the first time. (4) The magnitude of the behavior shift caused by stimulus familiarity seems to 460 depend upon the time elapsed between S1 and S2 (Fig. 4C). For now, we are limited to 461 speculation in order to explain the results (3) and (4). More study is needed to better understand 462 the cognitive processes involved.

463

464 Acknowledgements

465 Nicolas Brunet acknowledges grant support by the National Institutes of General Medical 466 Sciences (INBRE, P20 GM103476).

467

\section{References}

469

470

471

472

473
Akrami, A., Liu, Y., Treves, A., \& Jagadeesh, B. (2008). Converging neuronal activity in inferior temporal cortex during the classification of morphed stimuli. Cerebral Cortex, 19(4), 760776.

Anderson, B., Mruczek, R. E. B., Kawasaki, K., \& Sheinberg, D. (2008). Effects of familiarity on neural activity in monkey inferior temporal lobe. Cerebral Cortex, 18(11), 2540-2552. 
474 Bateson, M., Desire, S., Gartside, S. E., \& Wright, G. A. (2011). Agitated honeybees exhibit

475

476

477

478

479

480

481

482

483

484

485

486

487

488

489

490

491

492

493

494

495

496

497

498

499

500

501

502

503

504

505

506

507

508 pessimistic cognitive biases. Current Biology, 21(12), 1070-1073.

Begg, I. M., Anas, A., \& Farinacci, S. (1992). Dissociation of Processes in Belief: Source Recollection, Statement Familiarity, and the Illusion of Truth. Journal of Experimental Psychology: General. https://doi.org/10.1037/0096-3445.121.4.446

Blanchard, T. C., Wilke, A., \& Hayden, B. Y. (2014). Hot-hand bias in rhesus monkeys. Journal of Experimental Psychology: Animal Learning and Cognition, 40(3), 280.

Bonk, W. J., \& Healy, A. F. (2010). Learning and memory for sequences of pictures, words, and spatial locations: An exploration of serial position effects. American Journal of Psychology.

Bonnani, R. (2007). Primacy and recency effects in immediate free recall of sequences of spatial positions. Perceptual and Motor Skills. https://doi.org/10.2466/pms.105.6.483-500

Brunet, N. M., Bosman, C. A., Vinck, M., Roberts, M., Oostenveld, R., Desimone, R., ... Fries, P. (2014). Stimulus repetition modulates gamma-band synchronization in primate visual cortex. Proceedings of the National Academy of Sciences, 111(9), 3626-3631.

Burman, O. H. P., Parker, R. M. A., Paul, E. S., \& Mendl, M. T. (2009). Anxiety-induced cognitive bias in non-human animals. Physiology \& Behavior, 98(3), 345-350.

Dechêne, A., Stahl, C., Hansen, J., \& Wänke, M. (2010). The truth about the truth: A meta-analytic review of the truth effect. Personality and Social Psychology Review. https://doi.org/10.1177/1088868309352251

Erickson, C. A., Jagadeesh, B., \& Desimone, R. (2000). Clustering of perirhinal neurons with similar properties following visual experience in adult monkeys. Nature Neuroscience, 3(11), 1143.

Farrand, P., Parmentier, F. B. R., \& Jones, D. M. (2001). Temporal-spatial memory: Retrieval of spatial information does not reduce recency. Acta Psychologica. https://doi.org/10.1016/S0001-6918(00)00054-8

Fazio, L. K., Brashier, N. M., Keith Payne, B., \& Marsh, E. J. (2015). Knowledge does not protect against illusory truth. Journal of Experimental Psychology: General. https://doi.org/10.1037/xge0000098

Feng, S., Holmes, P., Rorie, A., \& Newsome, W. T. (2009). Can Monkeys choose optimally when faced with noisy stimuli and unequal rewards? PLoS Computational Biology. https://doi.org/10.1371/journal.pcbi.1000284

Freyd, J. J. (1983). The mental representation of movement when static stimuli are viewed. Perception \& Psychophysics. https://doi.org/10.3758/BF03202940

Gotts, S. J., Chow, C. C., \& Martin, A. (2012). Repetition priming and repetition suppression: A case for enhanced efficiency through neural synchronization. Cognitive Neuroscience, 3(34), 227-237. 
509 Grill-Spector, K., Henson, R., \& Martin, A. (2006). Repetition and the brain: neural models of $510 \quad$ stimulus-specific effects. Trends in Cognitive Sciences, 10(1), 14-23.

511 Harding, E. J., Paul, E. S., \& Mendl, M. (2004). Animal behaviour: cognitive bias and affective state. $512 \quad$ Nature, 427(6972), 312.

513 Herbranson, W. T., \& Schroeder, J. (2010). Are birds smarter than mathematicians? Pigeons 514 (Columba livia) perform optimally on a version of the Monty Hall Dilemma. Journal of $515 \quad$ Comparative Psychology, 124(1), 1.

516 Judge, S. J., Richmond, B. J., \& Chu, F. C. (1980). Implantation of magnetic search coils for measurement of eye position: An improved method. Vision Research.

518

519

520

521

522

523

524

525

526

527

528

529

530

531

532

533

534

535

536

537

538

539

540

541

542 https://doi.org/10.1016/0042-6989(80)90128-5

Kissinger, S. T., Pak, A., Tang, Y., Masmanidis, S. C., \& Chubykin, A. A. (2018). Oscillatory encoding of visual stimulus familiarity. Journal of Neuroscience, 38(27), 6223-6240.

Klein, E. D., Evans, T. A., Schultz, N. B., \& Beran, M. J. (2013). Learning how to "make a deal": Human (Homo sapiens) and monkey (Macaca mulatta) performance when repeatedly faced with the Monty Hall Dilemma. Journal of Comparative Psychology, 127(1), 103.

Lappe, M., Krekelberg, B., \& Lappe, M. (2000). The position of moving objects. Science.

Lazer, D. M. J., Baum, M. A., Benkler, Y., Berinsky, A. J., Greenhill, K. M., Menczer, F., ... Zittrain, J. L. (2018). The science of fake news. Science. https://doi.org/10.1126/science.aao2998

Liu, Y., \& Jagadeesh, B. (2008). Neural selectivity in anterior inferotemporal cortex for morphed photographic images during behavioral classification or fixation. Journal of Neurophysiology, 100(2), 966-982.

Liu, Y., Murray, S. O., \& Jagadeesh, B. (2009). Time course and stimulus dependence of repetitioninduced response suppression in inferotemporal cortex. Journal of Neurophysiology, 101(1), 418-436.

Mendl, M., Brooks, J., Basse, C., Burman, O., Paul, E., Blackwell, E., \& Casey, R. (2010). Dogs showing separation-related behaviour exhibit a 'pessimistic'cognitive bias. Current Biology, 20(19), R839-R840.

Murdock, Bennet B., J. (1962). The serial position effect of free recall. Journal of Experimental Psychology.

Nijhawan, R. (1994). Motion extrapolation in catching [9]. Nature. https://doi.org/10.1038/370256b0

Peissig, J. J., Singer, J., Kawasaki, K., \& Sheinberg, D. L. (2006). Effects of long-term object familiarity on event-related potentials in the monkey. Cerebral Cortex, 17(6), 1323-1334.

Roelofs, S., Boleij, H., Nordquist, R. E., \& van der Staay, F. J. (2016). Making decisions under 
543

544

545

546

547

548

549

550

551

552

553

554

555

556

557

558

559

560

561

562

563

564

565

566

567

568

569

570 ambiguity: judgment bias tasks for assessing emotional state in animals. Frontiers in Behavioral Neuroscience, 10, 119.

Sands, S. F., \& Wright, A. A. (1980). Primate memory: Retention of serial list items by a rhesus monkey. Science, 209(4459), 938-940.

Santiago, H. C., \& Wright, A. A. (1984). Pigeon memory: same/different concept learning, serial probe recognition acquisition, and probe delay effects on the serial-position function. Journal of Experimental Psychology: Animal Behavior Processes, 10(4), 498.

Shepard, R. N. (1984). Ecological constraints on internal representation: Resonant kinematics of perceiving, imagining, thinking, and dreaming. Psychological Review. https://doi.org/10.1037/0033-295X.91.4.417

Skinner, B. F. (1948). 'Superstition'in the pigeon. Journal of Experimental Psychology, 38(2), 168.

Tanaka, J. W., \& Curran, T. (2001). A neural basis for expert object recognition. Psychological Science, 12(1), 43-47.

Tremblay, S., Parmentier, F. B. R., Guérard, K., Nicholls, A. P., \& Jones, D. M. (2006). A spatial modality effect in serial memory. Journal of Experimental Psychology: Learning Memory and Cognition. https://doi.org/10.1037/0278-7393.32.5.1208

Vanderveldt, A., Oliveira, L., \& Green, L. (2016). Delay discounting: pigeon, rat, human-does it matter? Journal of Experimental Psychology: Animal Learning and Cognition, 42(2), 141.

Wang, W. C., Brashier, N. M., Wing, E. A., Marsh, E. J., \& Cabeza, R. (2016). On known unknowns: Fluency and the neural mechanisms of illusory truth. Journal of Cognitive Neuroscience. https://doi.org/10.1162/jocn_a_00923

Weiss, A. R., Guo, W., Richardson, R., \& Bachevalier, J. (2017). Intact perceptual ability, but impaired familiarity judgment, after neonatal perirhinal lesions in rhesus macaques. Developmental Cognitive Neuroscience, 28, 54-64.

Zhu, X. O., Brown, M. W., \& Aggleton, J. P. (1995). Neuronal Sianallina of Information Imoortant to Visual Recognition-Memory in Rat Rhinal aid Neighbouring Cortices. European Journal of Neuroscience, 7(4), 753-765. 


\section{Figure 1}

Examples illustrating the stimuli and experimental paradigm.

(A) For each session, all stimuli were derived from one pair of images selected from a picture data set (see black boxes for some examples of stimuli pairs). (B) Dynamic cues were drawn from a larger 11-frames animation, generated by morphing both images of an image pair into each other. Static cues were identical to the images of the choice pair (see second row), while dynamic cues were formed by the first five (movie A, blue box) or last five (movie B, pink box) frames of the larger movie. Ambiguous dynamic cues were obtained by selecting the five frames from the middle (movie M, green box). The playing direction of trials with dynamic cue was determined randomly (see both rows in blue, pink and green boxes). Weighted cues were generated by skipping the penultimate frame (see frames marked with a red crossed box), which resulted in a 100 ms gap during the display of the cue. (C) Schematic representation of a trial: First, a cue, either static (image A or image B) or dynamic (movie A, B, or M), was displayed. The color of the boxes in (C) matches the examples shown in (B). Targets, leading to reward, are marked with a small square $(100 \%)$ or small triangle (50\%, randomly determined), and colored to match the cue types shown in the colored boxes. 


\section{Brunet and Jagadeesh, Figure 1}

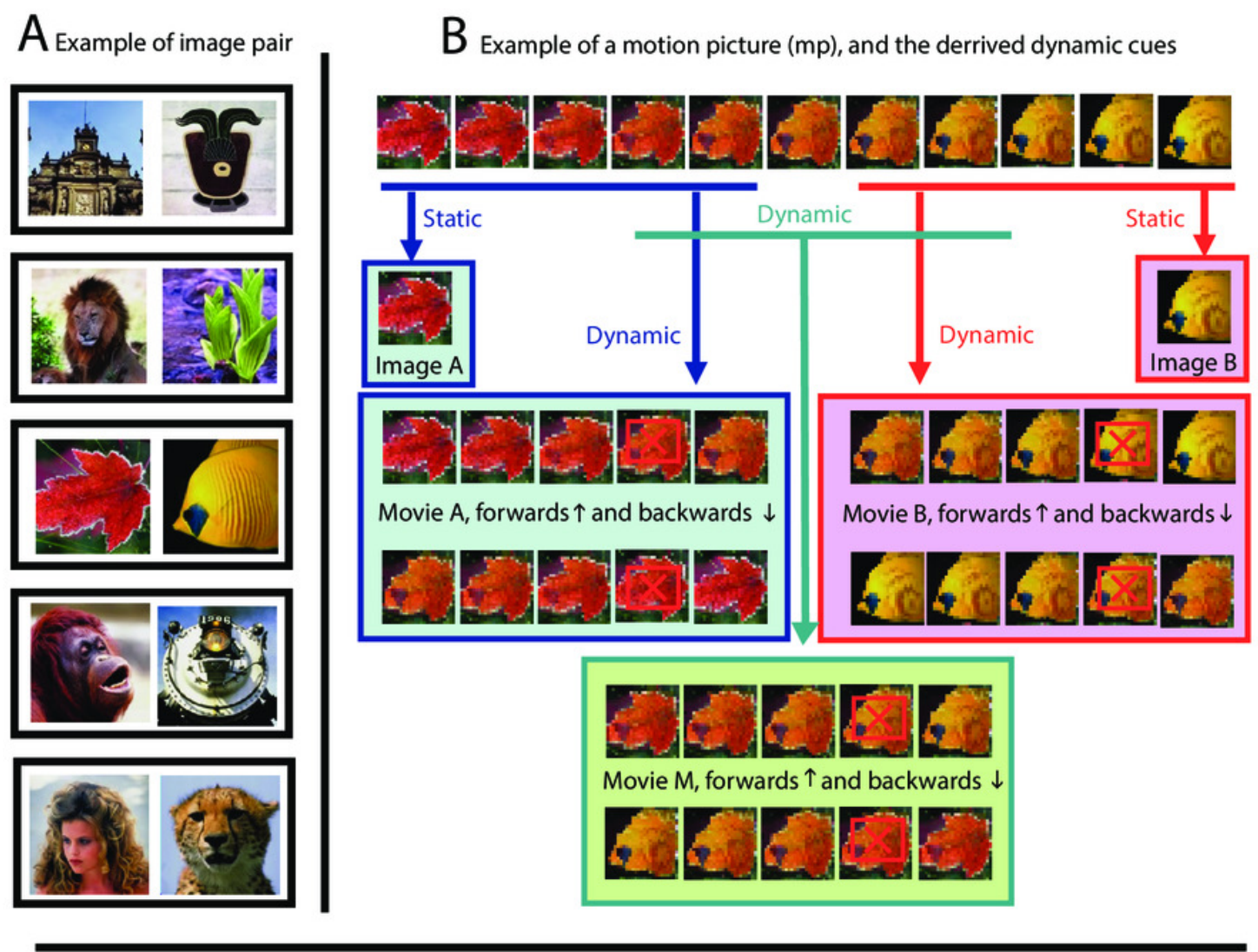

C Order of events, illustrated with example

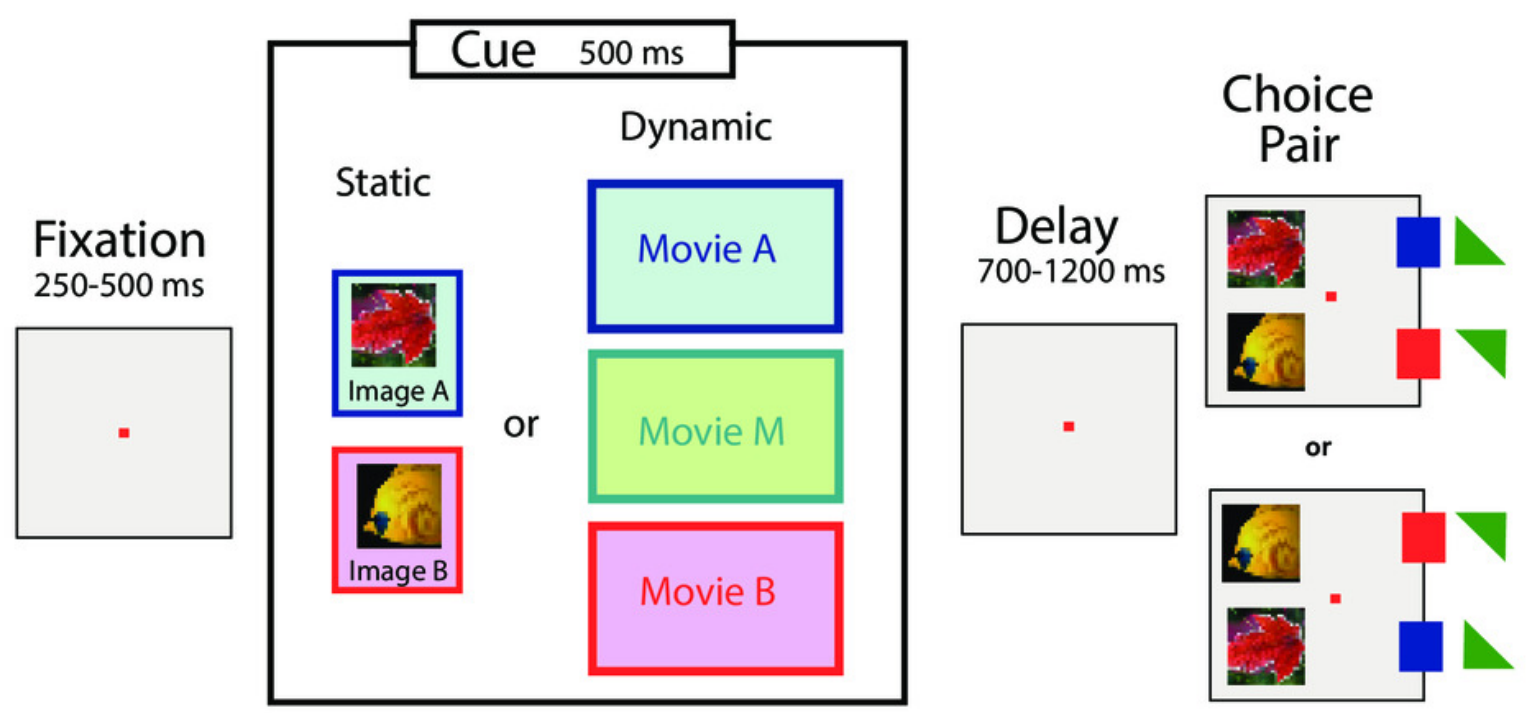




\section{Figure 2}

Analysis reward ratios for each NHP and each experimental condition.

(A and B) Percentage of rewarded trials for different dynamic cue conditions. Conditions are represented with different colors: Purple for unambiguous trials played in the forward direction (for an example, see Fig. 1B, first row in blue box or second row in pink box). Green for unambiguous trials played in the reverse direction (for an example, see Fig. 1B, second row in blue box or first row in pink box). Gray for ambiguous trials in either direction (for examples, see Fig. 1B, green box). The results were computed by averaging performance across all sessions; the error bars denote SE. (C and D) Percentage of rewarded trials as a function of normalized trial number for different conditions (colors match conditions in panels $A$ and $B$ ). The results were obtained by first normalizing each session with respect to trial number and condition, and then averaging across all sessions. 


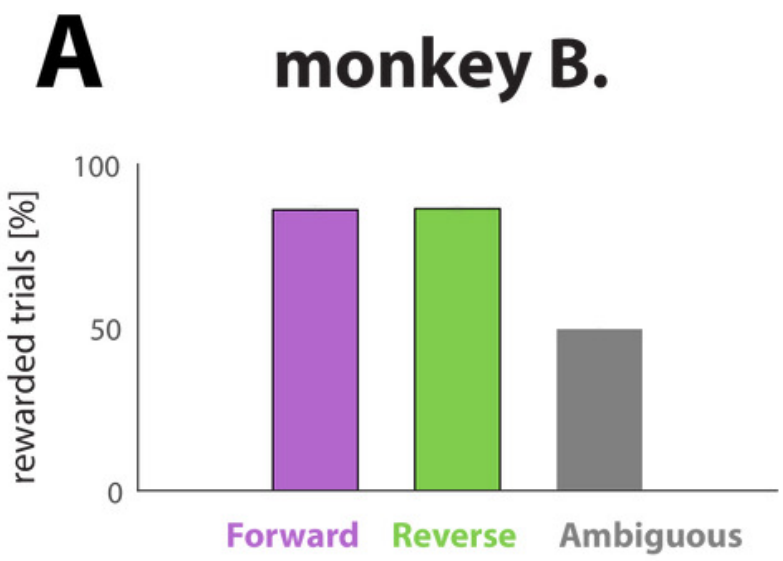

B monkey S.
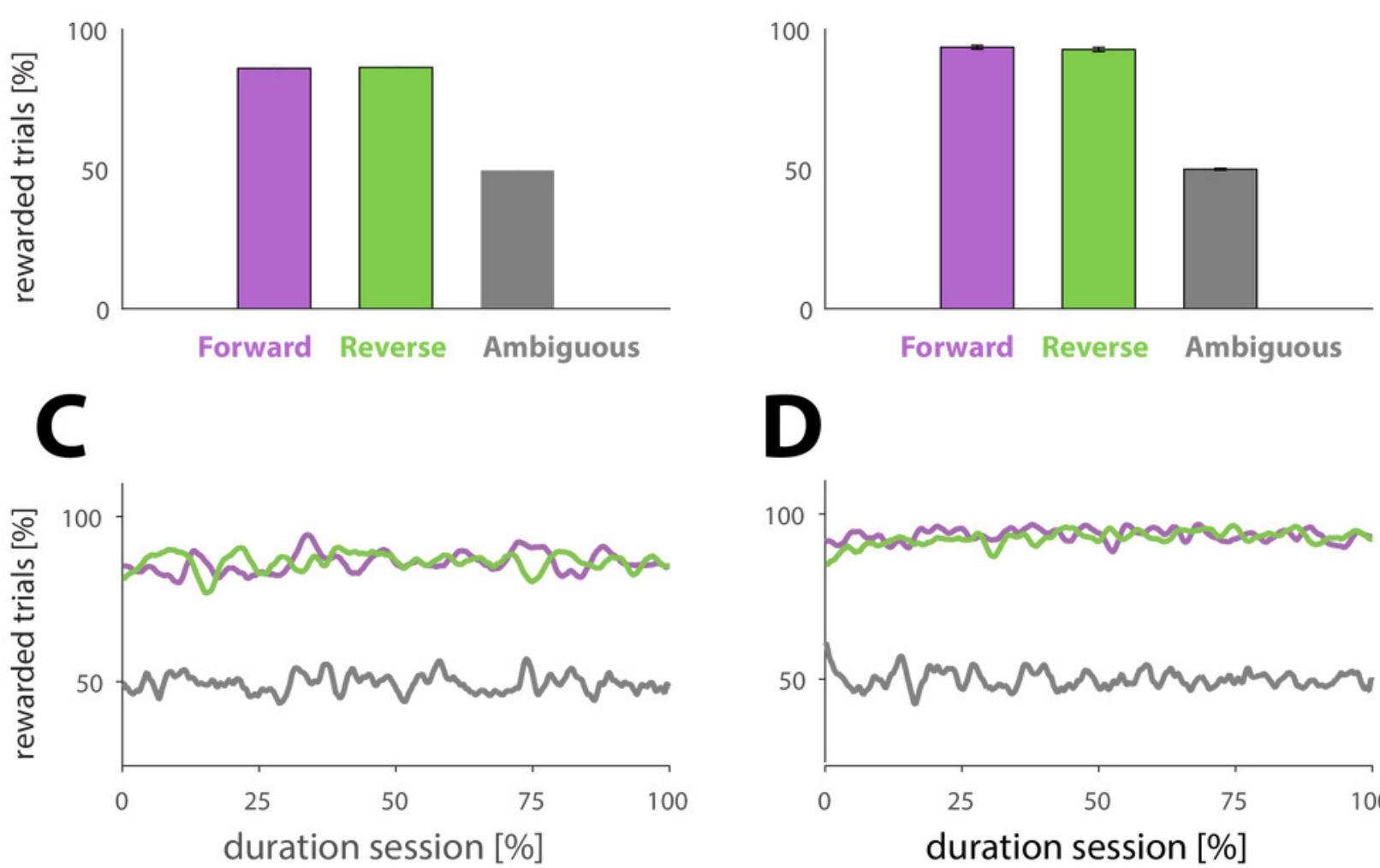
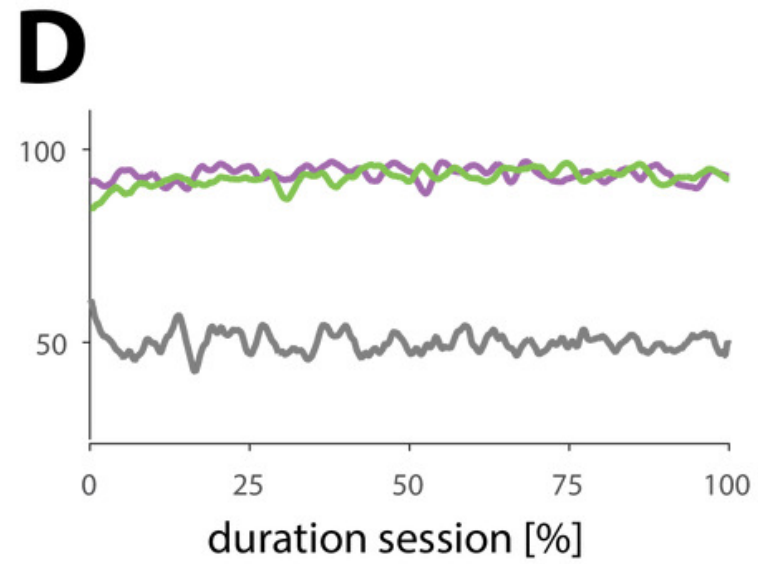


\section{Figure 3}

Analysis behavior for trials with ambiguous dynamic cues.

(A and B) Histrogram showing the distribution of PI values computed for each session (see methods). A PI of zero (marked with vertical line) represent sessions where behavior was equally balanced between 'recency' and 'primacy'. Values are further shifted away from zero (negative for primacy and positive for recency) with bias strength. The insets show the PI value, averaged across all sessions for each primate; error bars denote SE. (C and D).

Behavior for trials with ambiguous dynamic cue as a function of normalized trial number. Each session was normalized with respect to trial number. C and D show the average across all normalized sessions.
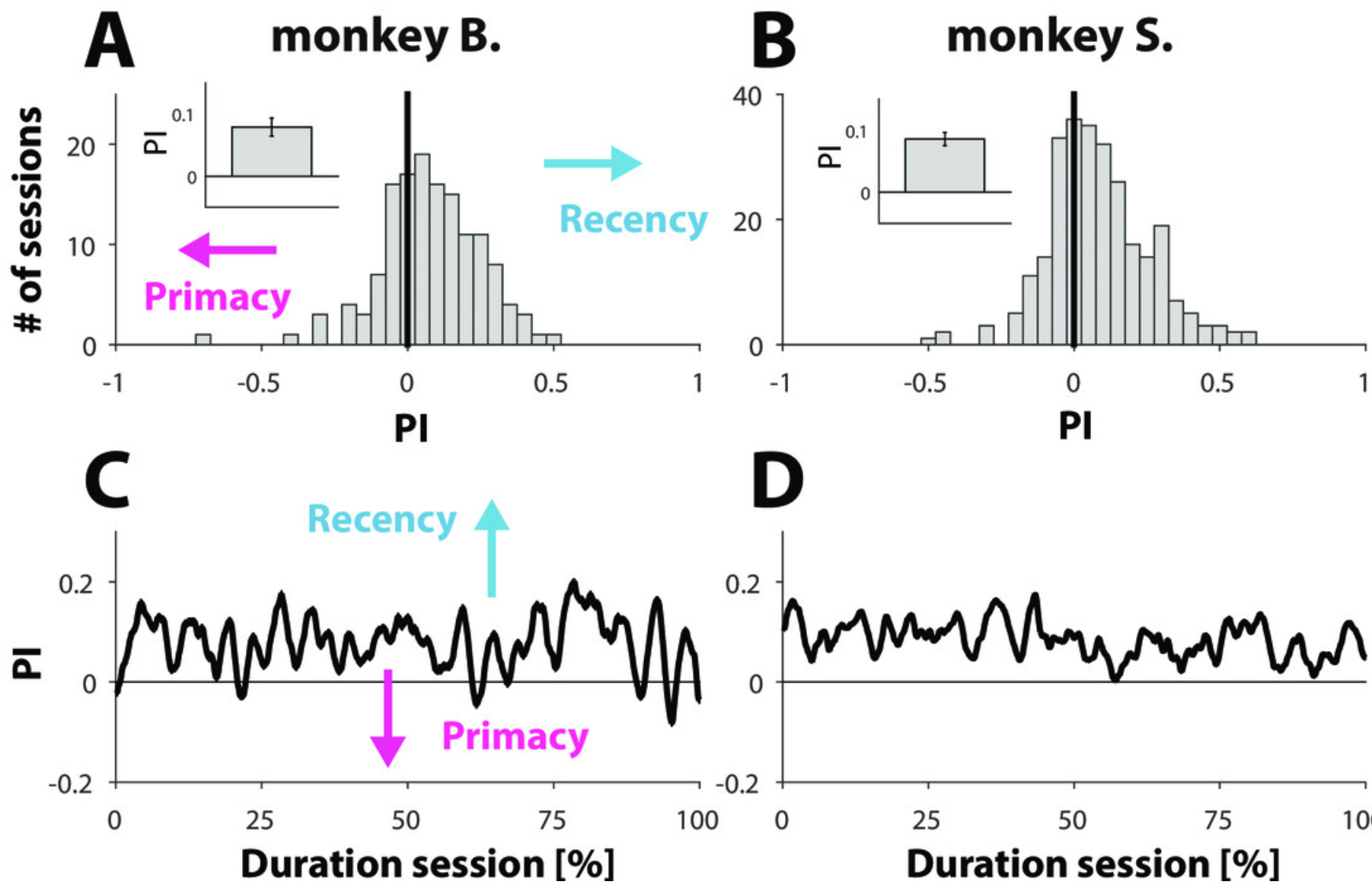

D

PI

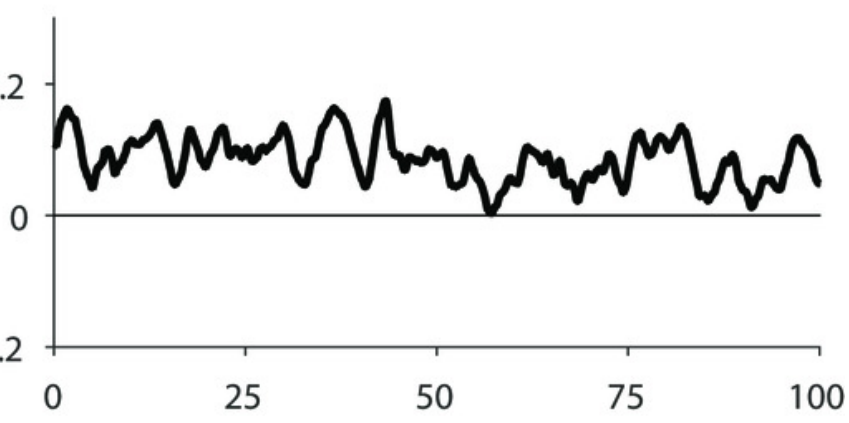

Duration session [\%] 


\section{Figure 4}

\section{Effect of stimulus familiarity.}

(A and B) Average PI value (see methods) averaged across all S1 sessions (visual stimuli are novel) and all S2 sessions (visual stimuli are familiar). A PI value of zero is marked with a horizontal line. Only sessions where an S1-S2 pair (matching stimuli) could be formed are included in the analysis. Throughout all panels the colors green and purple are used to indicate data and results respectively from monkeys B. and $\mathrm{S}$. (C) Scatterplot where each circle represents one S1-S2 pair, with X and $Y$ coordinates corresponding to the PI values respectively computed for the S1 and S2 session of that pair. Datapoints on top or close to the diagonal line indicate pairs of sessions where familiarity with the stimuli had little or no effect. Note that most datapoints fall into the upper triangle, consistent with a shift towards greater recency bias with stimulus familiarity. (D) Averaged $\Delta_{\mathrm{Pl}}$ (see methods) across all S1-S2 pairs, split into 3 bins according to time elapsed between S1 and S2. Note that $\Delta_{\mathrm{Pl}}$ ranges from -1 to 1, but that all values shown in $\mathrm{D}$ are above zero.

Error bars in panels A,B and D denote SE. Asterisks (*) indicate statistical significance between S1 and S2 (one, two or three asteriks indicate $p$-values that are respectively smaller than $0.05,0.01$, and 0.001 ). 

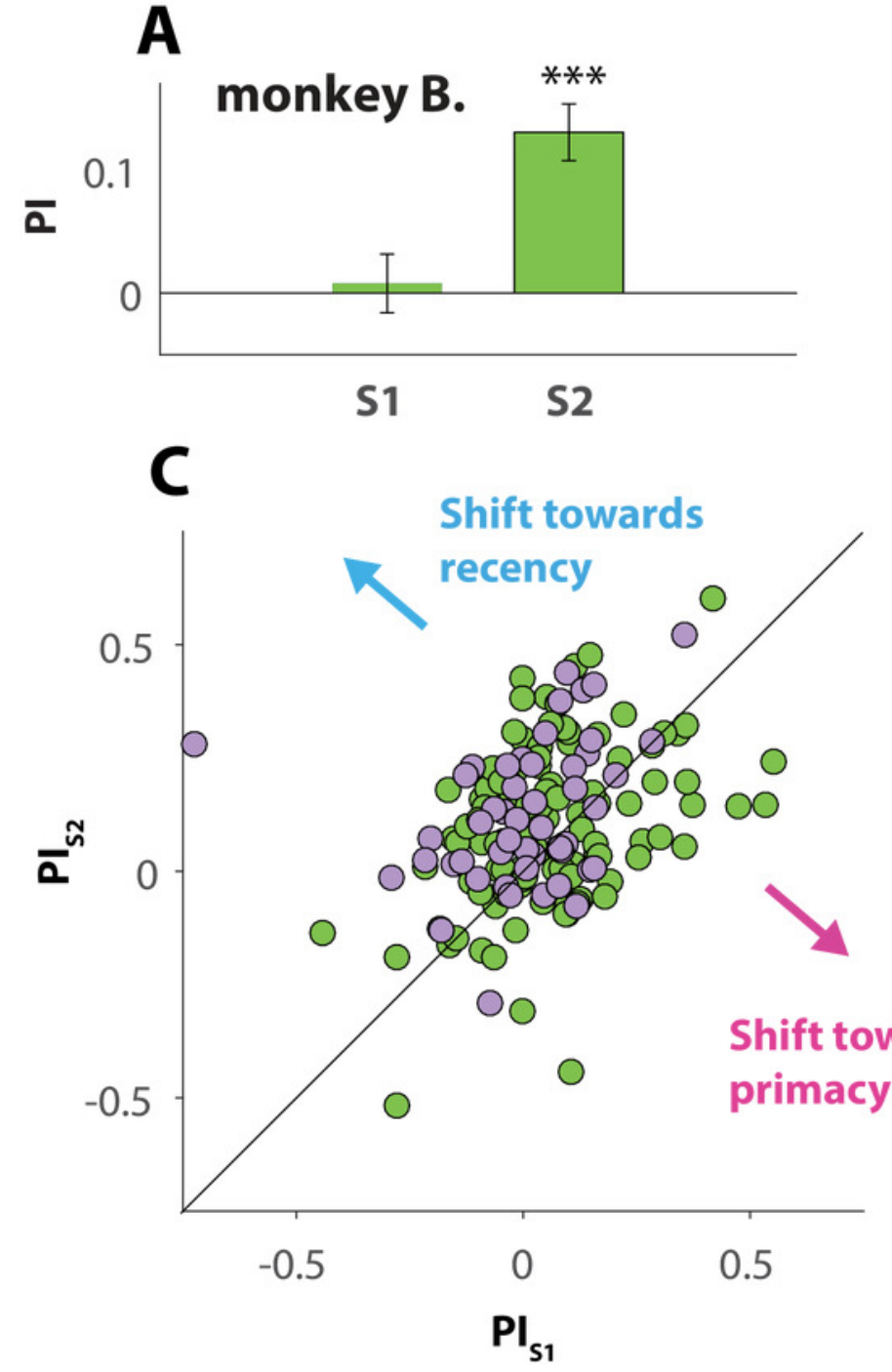

B

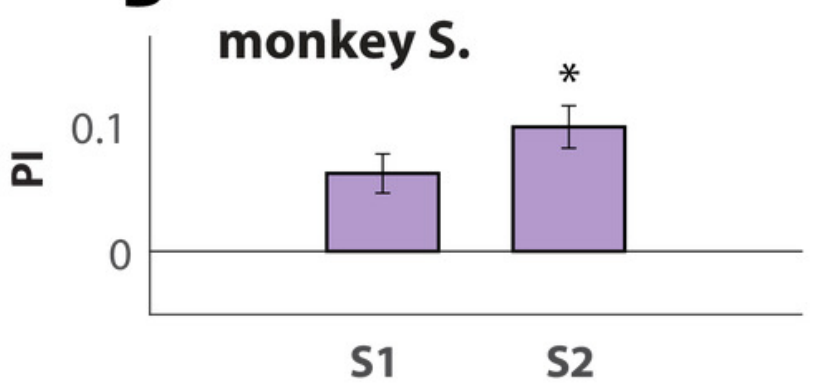

D

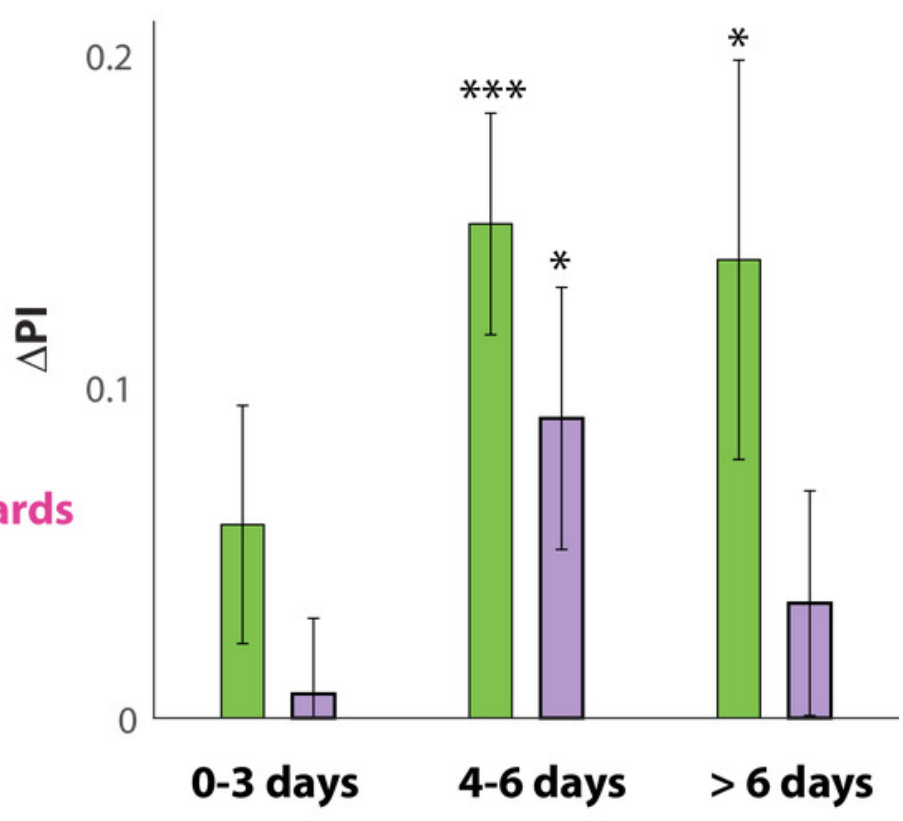




\section{Figure 5}

Changes in behavior across session and effect of reinforcement history.

(A and B) Pl values as a function of recording day. Sessions are thus in chronological order. The black lines represent linear regression lines. For illustration purpose, one pair of S1 and S2 was marked in each panel. The parent stimuli for those sessions are shown as insets. (C and D) $\triangle \mathrm{PI}$ as a function of reward frequency during performance of $\mathrm{S1}$, for trials where the primates selected the primacy option (C) or the recency option (D). Green (monkey B.) and purple (Monkey S.) lines represent the regression slopes for each dataset. The insets illustrate the expected correlation for behavior affected by a strong effect of reinforcement history. 

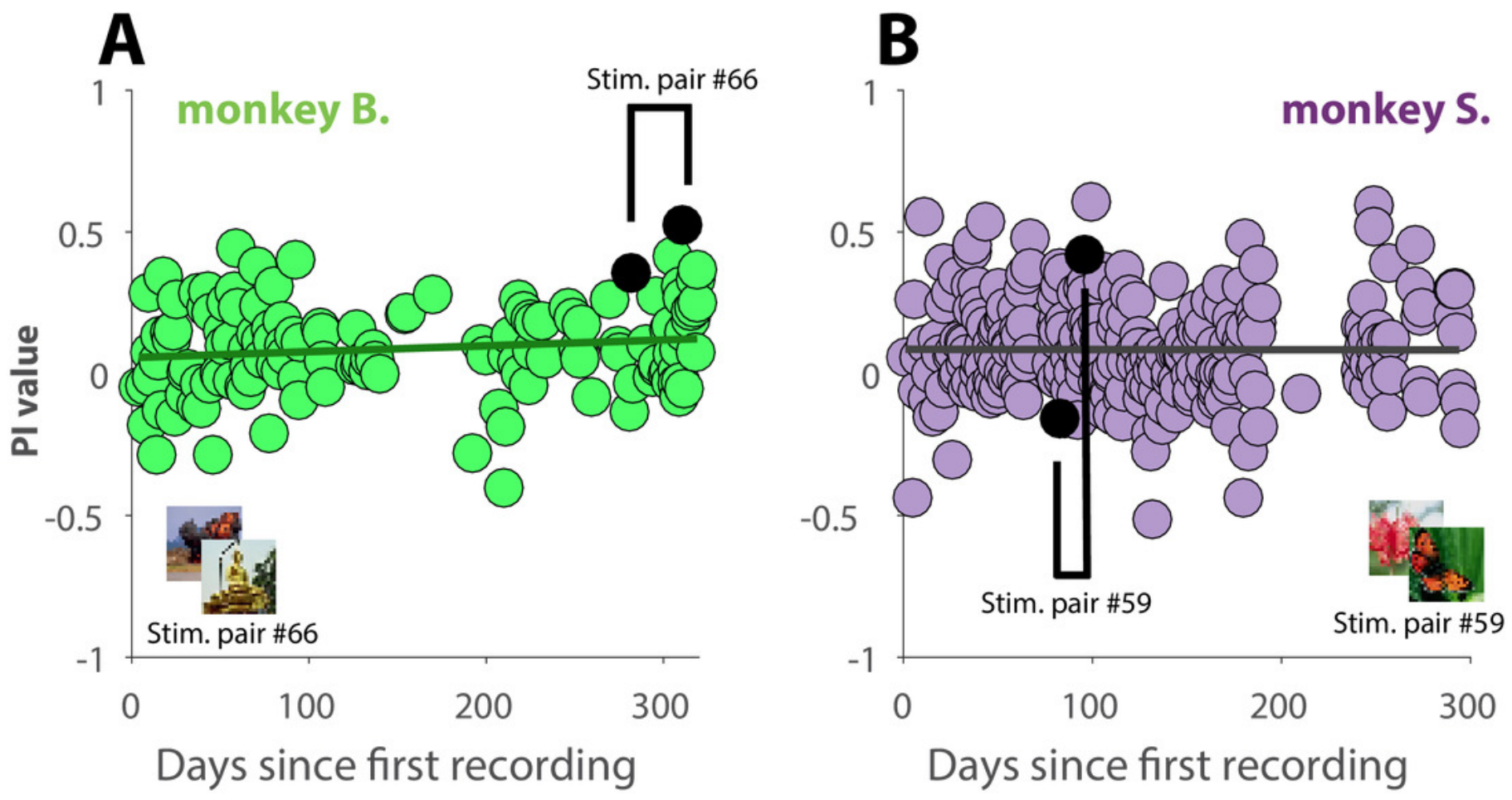

When choosing "primacy"
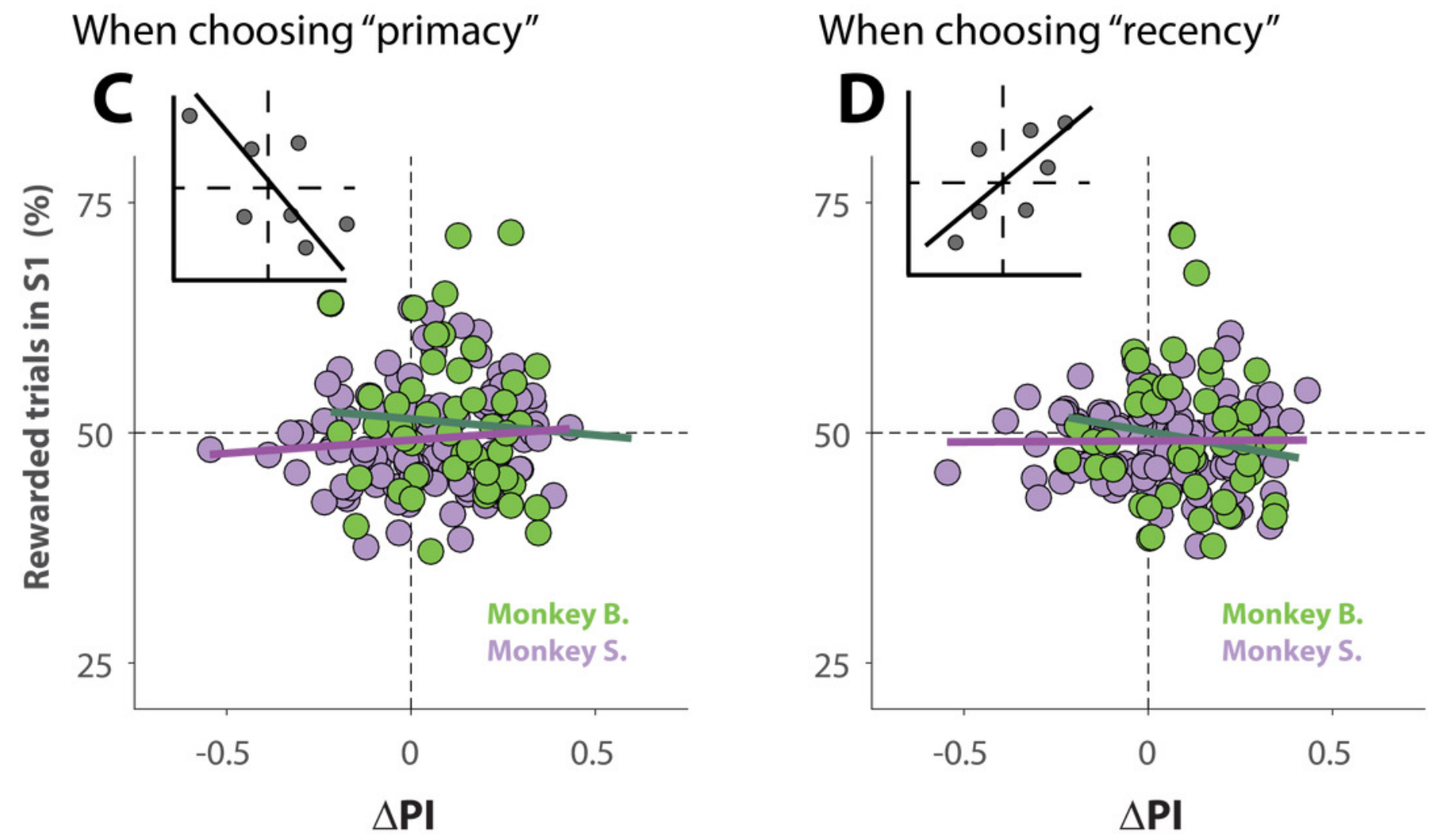